\title{
Treatment of Rectal Cancer-Induced Disseminated Carcinomatosis of the Bone Marrow with FOLFOX plus Cetuximab and Panitumumab
}

\author{
Takehito Ehara Masato Kitazawa Nao Hondo Shugo Takahata \\ Yuta Yamamoto Makoto Koyama Motohiro Okumura \\ Satoshi Nakamura Shigeo Tokumaru Futoshi Muranaka \\ Yusuke Miyagawa Yuji Soejima \\ Department of Surgery, Shinshu University School of Medicine, Matsumoto, Japan
}

\begin{abstract}
Keywords
Disseminated carcinomatosis of the bone marrow - Cetuximab - Colorectal cancer .

Panitumumab
\end{abstract}

\section{Abstract}

Disseminated carcinomatosis of the bone marrow (DCBM) in colorectal cancer is an extremely rare complication with a poor prognosis. Here, we report a case of DCBM due to rectal cancer successfully treated with a combination of FOLFOX and an anti-epidermal growth factor receptor (EGFR) agent. The patient was a 38-year-old man diagnosed with rectal cancer with multiple bone and para-aortic lymph node metastases complicated by disseminated intravascular coagulation (DIC). He first recovered from DIC following cotreatment with FOLOX plus cetuximab; subsequently, the second attack was successfully treated with FOLFOX plus panitumumab. His initial condition was extremely poor, but he survived with two FOLFOX plus anti-EGFR regimens and died 333 days after introduction of chemotherapy.

\section{Introduction}

Disseminated carcinomatosis of the bone marrow (DCBM) is characterized by diffuse organ infiltration centered on the bone marrow and is a type of cancer metastasis frequently associated with disseminated intravascular coagulation (DIC) [1, 2]. Most primary lesions associated with DCBM are observed in gastric, prostate, and breast cancer, whereas it is rare 


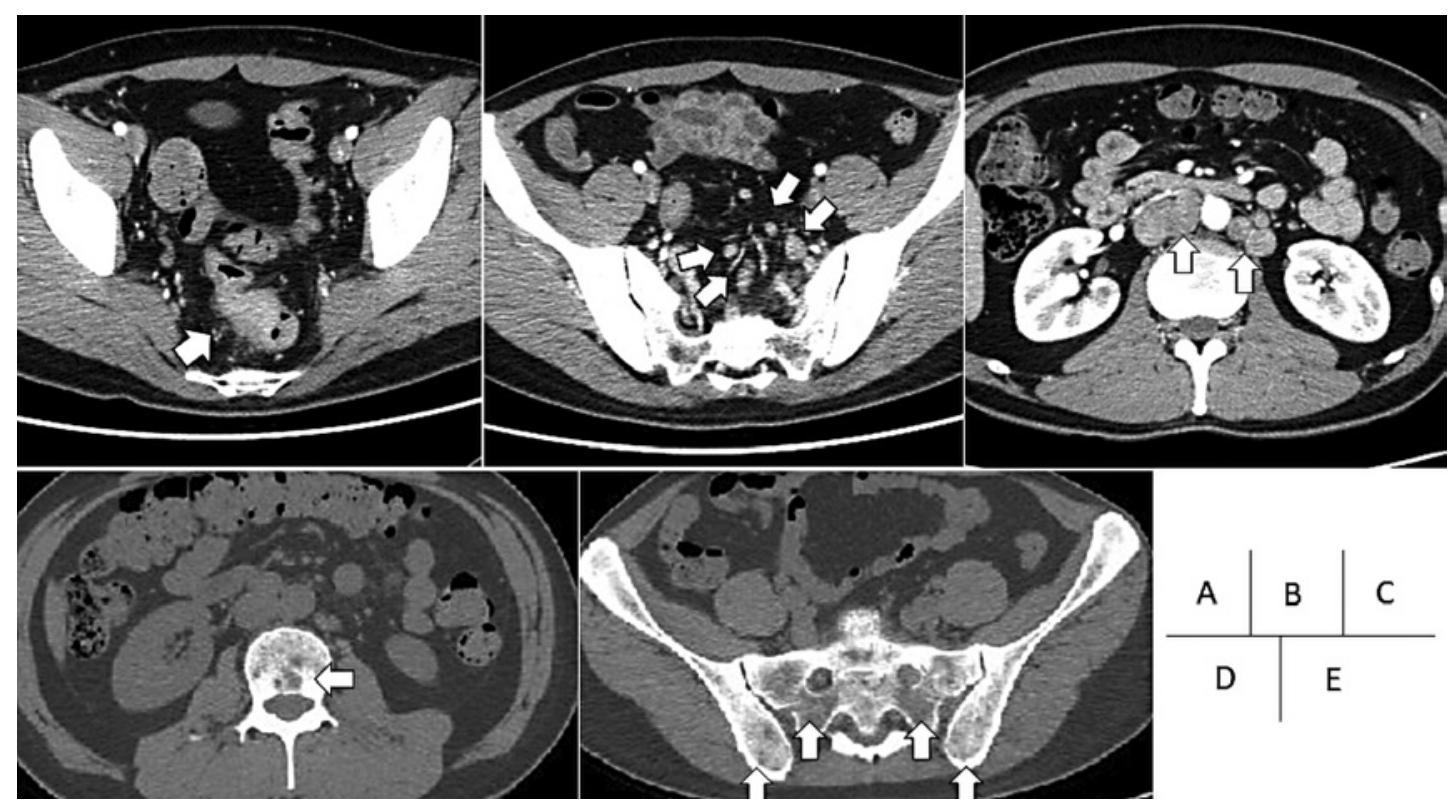

Fig. 1. A CT shows wall thickness with contrast effect in the upper rectum (arrow). B, C CT shows multiple lymph node enlargement beside the rectum (arrows in B) and in the para-aortic lesion (arrows in C). D, E CT shows osteolytic changes in multiple bones including the vertebrae (arrows in D), sacrum, and iliac bones (arrows in E).

in colorectal cancer $[3,4]$. The prognosis of DCBM without chemotherapy is reported to be extremely poor [5].

Herein, we report a case of DCBM due to rectal cancer successfully treated with FOLFOX plus an anti-epidermal growth factor receptor (EGFR) agent, cetuximab (Erbitux ${ }^{\circledR}$, Merck Biopharma, Darmstadt, Germany), as a first-line chemotherapy and FOLFOX plus another anti-EGFR agent, panitumumab (Vectibix ${ }^{\circledR}$, Takeda Pharmaceutical, Tokyo, Japan), as the fourth-line chemotherapy.

\section{Case Report}

The patient was a 38-year-old man who had previously visited another hospital because of pain in his lower back and lower extremities. His Eastern Cooperative Oncology Group (ECOG) performance status was 3 and his axillary temperature was $38.9^{\circ} \mathrm{C}$. There was no abnormal finding in the abdomen or subcutaneous hemorrhage. Blood test results revealed a decreased red blood cell count, hemoglobin level, and platelet count $-380 \times 10^{4} / \mu \mathrm{L}, 10.6$ $\mathrm{g} / \mathrm{dL}$, and $12.5 \times 10^{3} / \mu \mathrm{L}$, respectively - while the white blood cell count increased to $12,600 /$ $\mu \mathrm{L}$. Analysis of the patient's coagulation system revealed a prolonged prothrombin time/ international normalization ratio (14.0 s/1.23) and increased levels of fibrinogen (491 $\mu \mathrm{g} /$ $\mathrm{mL})$ and D-dimer $(90.9 \mu \mathrm{g} / \mathrm{mL})$. Serum biochemistry test results revealed elevated levels of lactate dehydrogenase (LDH) at 5,850 IU/L, alkaline phosphatase (ALP) at 3,475, and aspartate aminotransferase (AST), alanine aminotransferase (ALT), and C-reactive protein (CRP) at 92, 336, and $28.13 \mathrm{mg} / \mathrm{dL}$, respectively. The serum level of carcinoembryonic antigen (CEA) was elevated at $13.6 \mathrm{ng} / \mathrm{mL}$. The CT showed wall thickness with contrast effect located in the upper rectum (Fig. 1A) and multiple lymph node enlargements beside the rectum (Fig. 1A) and in the para-aortic lesion (Fig. 1C). Furthermore, the CT showed osteolytic 


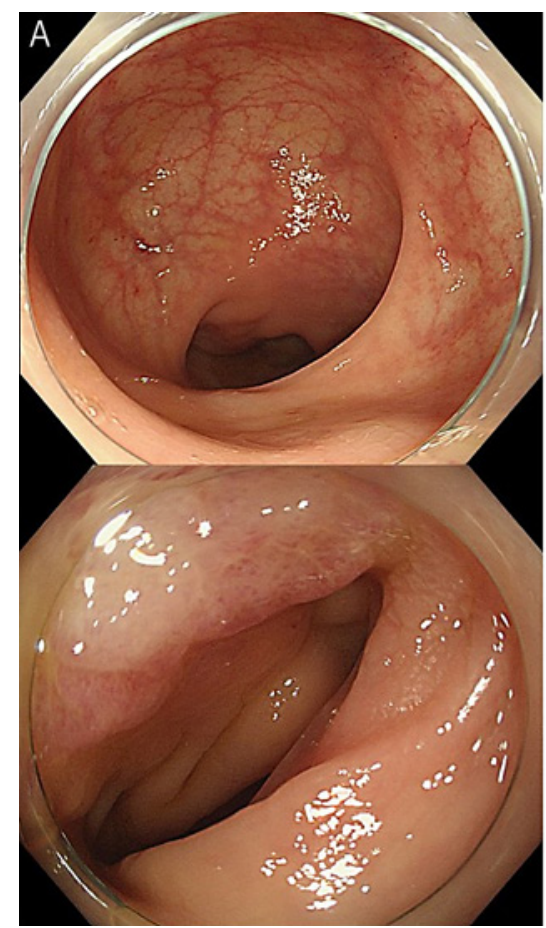

B
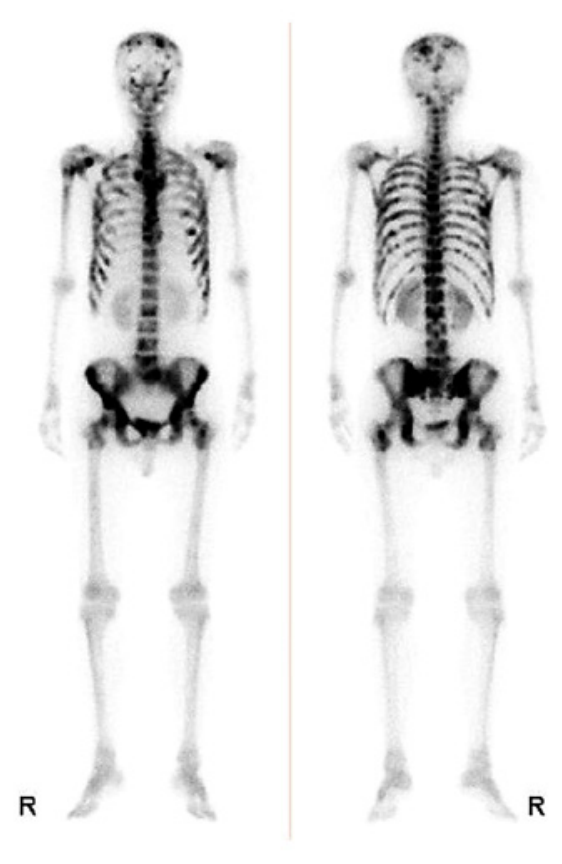

Fig. 2. A Colonoscopy shows elevated lesion in the upper rectum without stenosis. B Bone scintigraphy shows abnormal diffuse accumulation in the cranial bone, spine, shoulder blade, right clavicle, and pelvic bone, and bilaterally in ribs and femur.

changes in multiple bones including the sacrum, ilium, and vertebrae (Fig. 1D,E). The colonoscopy showed elevated lesion in the upper rectum without stenosis (Fig. 2A). A tumor biopsy specimen from the patient displayed a poorly differentiated adenocarcinoma with a mucinous component. Bone scintigraphy revealed abnormal diffuse accumulation in the cranial bone, spine, shoulder blade, right clavicle, and pelvic bone and bilaterally in the ribs and femur (Fig. 2B). The patient was diagnosed with diffuse bone marrow metastasis originating from rectal cancer.

The patient's condition was very poor with platelet counts declining $>30 \%$ within $24 \mathrm{~h}$ meeting the criteria of acute DIC. However, the patient's condition did not meet the criteria for a safe initiation of chemotherapy because his performance status was 3 , and he had thrombopenia, and high AST and ALT levels. Capecitabine plus oxaliplatin (CapeOX) was administered without dose reduction along with recombinant human-soluble thrombomodulin (Recomodulin ${ }^{\circledR}$, Asahi Kasei Pharma, Tokyo, Japan). The patient's symptoms gradually improved and his abnormal laboratory findings of D-dimer, ALP, LDH, and CEA levels rapidly decreased with the treatment; he recovered from the DIC (Fig. 3).

The RAS gene was identified as wild type; therefore, the second chemotherapy regimen was changed to FOLFOX plus cetuximab and, subsequently, the CT showed markedly reduced para-aortic lymph nodes (Fig. 4A, B). After the eighth course of FOLFOX plus cetuximab, the patient developed a high fever, the lower back pain recurred, and the CT showed enlarged para-aortic lymph nodes. The patient was administered two courses of FOLFIRI plus aflibercept and one course of regorafenib, but neither had any effect. The CT showed increased para-aortic lymph node metastasis (Fig. 4C), the blood test results indicated that his condition had worsened, and DIC recurred. Three treatment options, i.e., TAS102 (Lonsurf ${ }^{\circledR}$, Taiho 


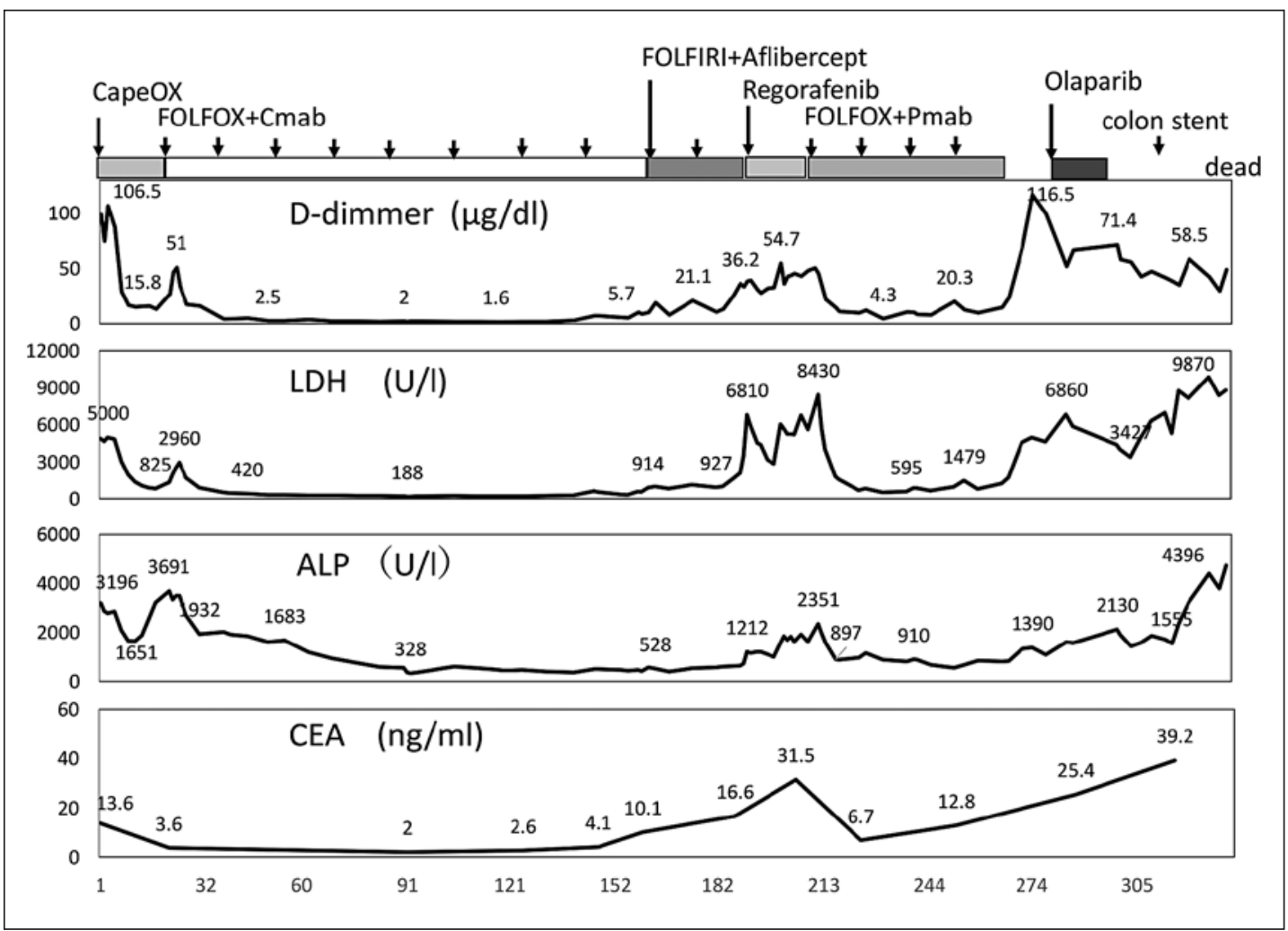

Fig. 3. Clinical course of this patient including chemotherapy and clinical data.

pharma, Tokyo, Japan), FOLFOX plus panitumumab, and best supportive care, were proposed to the patient, and he selected FOLFOX plus panitumumab.

Subsequently, the patient's condition improved again, and he recovered from the DIC after one course of FOLFOX plus panitumumab. After two courses of chemotherapy, his CT showed para-aortic lymph node shrinkage (Fig. 4D) and decreased tumor marker levels (Fig. 3). However, after four courses, the patient became septic with high fever and back pain. His CT showed a retroperitoneal abscess, enlarged para-aortic lymph nodes, and mediastinal lymph nodes metastasis.

We searched for cancer gene mutations using next-generation sequencing (tumorprofiling multiplex gene panels) and the results revealed mutations in the breast cancer (BRCA) gene. Based on the test result, olaparib (Lynparza ${ }^{\circledR}$, AstraZeneca, Cambridge, UK) was administered orally for 2 weeks; however, tumor marker levels were persistently elevated and the CT revealed no changes in the metastatic lymph node lesions. His condition rapidly worsened and he died 333 days after the initiation of chemotherapy.

\section{Discussion}

The relationship between bone metastasis of malignant tumors and hematological abnormalities is well known. Jarcho [5] first reported diffusely infiltrative scirrhous gastric cancer characterized by diffuse bone marrow metastasis, anemia, thrombocytopenia, and bleeding tendency in 1936. Brain et al. [6] demonstrated the relationship between mucin-producing adenocarcinoma, DIC, and microangiopathic hemolytic carcinoma in 1970. 


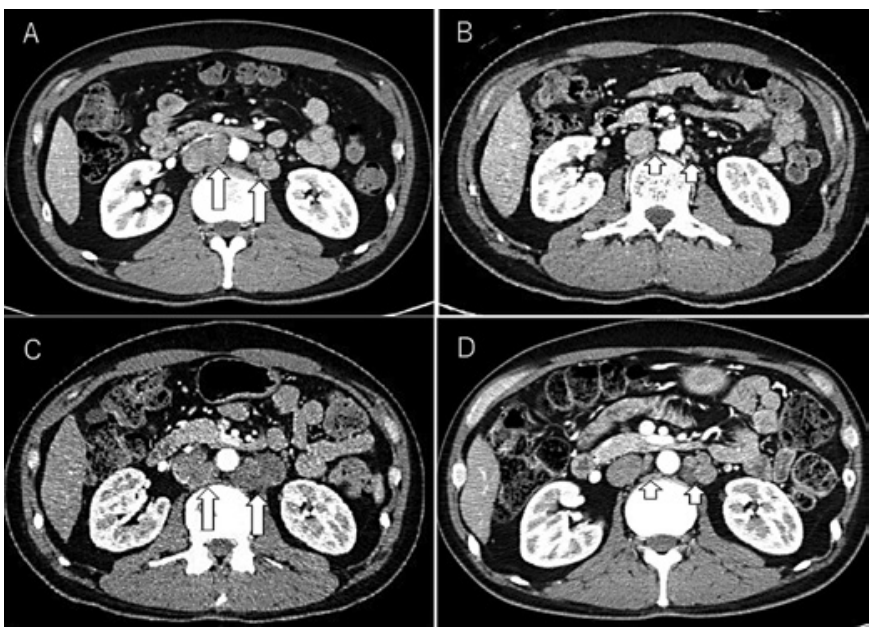

Fig. 4. A CT shows multiple enlargement of para-aortic lymph nodes before chemotherapy (long arrows). B After one course of capecitabine plus oxaliplatin (CapeOX) and three courses of FOLFOX plus cetuximab, the para-aortic lymph nodes shrank remarkably (short arrows). C Before FOLFOX plus panitumumab, CT showed re-enlarged para-aortic lymph nodes (long arrows). D After two courses of FOLFOX plus panitumumab, para-aortic lymph nodes shrank (short arrows).

Hayashi et al. [7] reported 40 cases of patients who presented with diffuse bone marrow metastasis and defined DCBM as a wide range of hematogenous and lymphatic metastases, causing bleeding tendencies due to DIC and hemolytic anemia. In that study, 37 of the 40 cases $(92.5 \%)$ were due to gastric cancer while one each was due to prostate, gallbladder, and ovarian cancer with unknown primary lesion, but no case of colorectal cancer was reported. A review of the literature revealed that DCBM in colorectal cancer is particularly rare and only 10 cases have been reported previously (Table 1) [8-17]. The average age of the patients was 61.0 years (men, 9 cases; women, 2 cases). The primary tumor was commonly located in the rectum $(n=6,54.5 \%)$, sigmoid colon $(n=2)$, ascending $(n=1)$, transverse $(n=1)$, and right side (no detailed site was noted) of the colon $(n=1)$.

The histological subtypes were signet ring-like cell adenocarcinoma $(n=3)$, poorly differentiated adenocarcinoma $(n=3)$, mucinous adenocarcinoma $(n=2)$, moderately differentiated adenocarcinoma $(n=2)$, and well differentiated $(n=1)$. The peripheral blood test findings revealed a low average platelet count $\left(9.1 \times 10^{4} / \mu \mathrm{L}\right)$. Generally, anticoagulation therapy, blood transfusion, and protease inhibitors have been used to treat DIC. Chemotherapy is usually not feasible in patients with DCBM because of their poor general condition at diagnosis with anemia, thrombocytopenia, elevated AST and ALT, and DIC. No chemotherapy was administered to 3 of 11 patients, with a mean survival time (MST) of only 15.6 days.

In contrast, 8 patients who had received some chemotherapy showed a significantly longer MST (273.5 days). Seven patients who had received chemotherapy with oxaliplatin (FOLFOX/CapeOX) showed an MST of 305.3 days. Four patients who received moleculartargeted agents (bevacizumab, cetuximab, and panitumumab) had prolonged survival time (377.0 days) compared with those who did not receive molecular-targeted agents (196.0 days). Our patient was successfully treated with FOLFOX plus an anti-EGFR agent, cetuximab, as the first-line chemotherapy, followed by reintroduction of FOLOFOX plus another antiEGFR agent, panitumumab, as the fourth-line chemotherapy. To the best of our knowledge, 


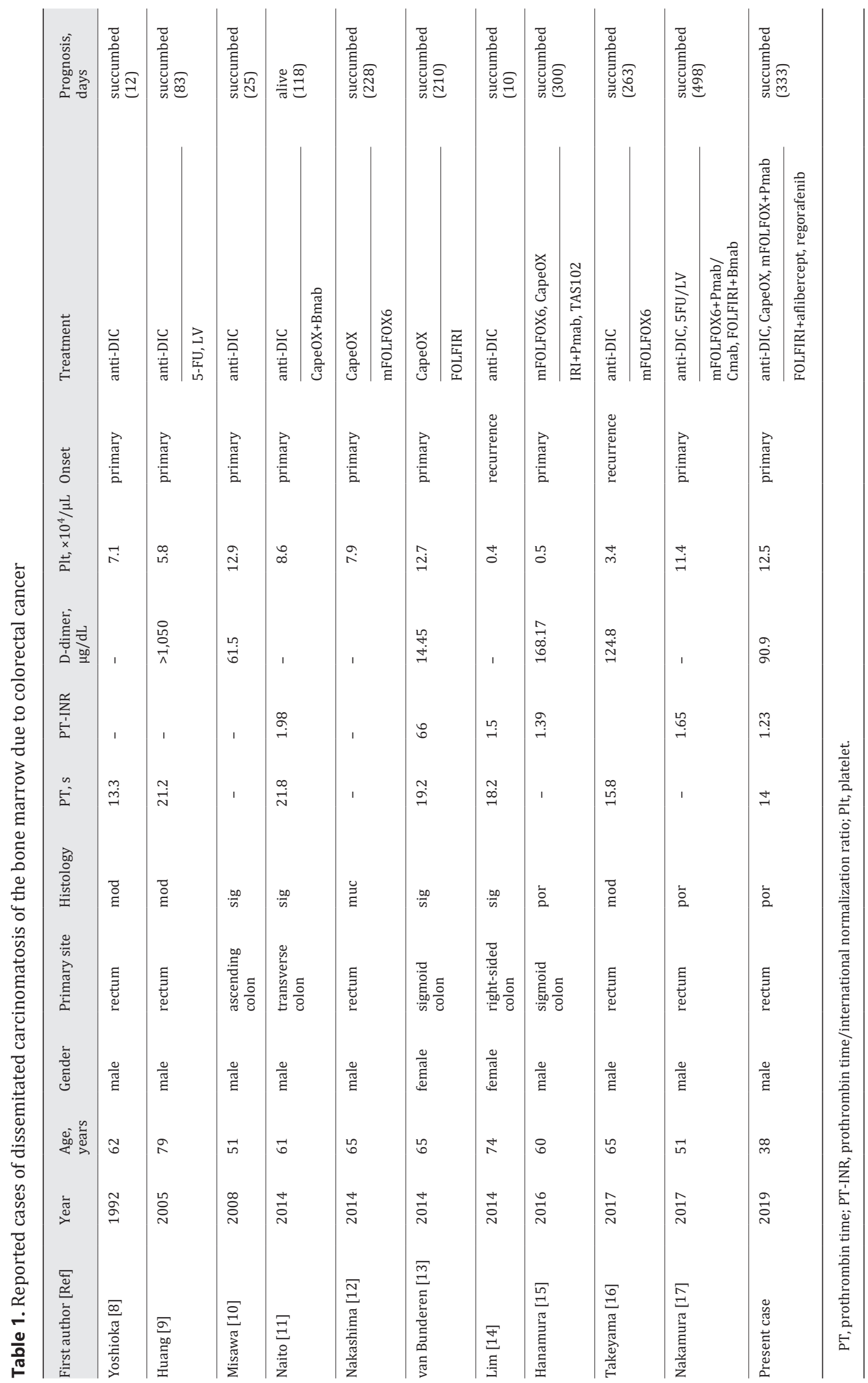


this is likely the first case report of reintroduction of FOLFOX plus an anti-EGFR agent as a successful treatment of DCBM.

The RE-OPEN study demonstrated the effect of reintroduction of oxaliplatin in patients with advanced colorectal cancer previously treated with oxaliplatin and irinotecan [18]. Reintroduction of anti-EGFR antibodies has also been reported to be effective [19, 20]. However, effective reintroduction of oxaliplatin and anti-EGFR agents has been limited to patients treated for a duration of 6 months because of discontinuation and reintroduction in these reports. Recently, the REVERCE Study demonstrated that anti-EGFR treatment after regorafenib prolonged the overall survival more than the current standard sequence of treatment with an anti-EGFR agent followed by regorafenib [21]. Furthermore, preclinically, Napolitano et al. [22] reported that regorafenib overcame anti-EGFR antibody resistance of colorectal cancer by blocking the mitogen-activated protein kinase (MAPK) and AKT pathways. In this present case, pretreatment with regorafenib may have overcome the resistance to the antiEGFR agents.

\section{Conclusion}

This case study revealed that FOLFOX plus anti-EGFR agents was effective in a patient with colorectal cancer with DIC. Therefore, reintroduction of FOLFOX plus an anti-EGFR agent may be a feasible treatment option for this class of patients.

\section{Statement of Ethics}

Written ethical approval for the publication on the present case report was obtained from the patient.

\section{Disclosure Statement}

The authors declare that they have no conflicts of interest to disclose.

\section{Funding Sources}

No funding was received for this article and the authors have no conflicts of interest directly relevant to this report.

\section{Author Contributions}

All authors were involved in the preparation of the manuscript. Takehito Ehara, Nao Hondo, Shugo Takahata, Yuta Yamamoto, and Makoto Koyama collected the data, and Masato Kitazawa, Yusuke Miyagawa, and Yuji Soejima wrote the manuscript. Shugo Takahata, Yuta Yamamoto, Makoto Koyama, Motohiro Okumura, Nakamura Satoshi, Shigeo Tokumaru, and Futoshi Muranaka were involved in the treatment of the patient. All authors read and approved the final manuscript. 
Ehara et al.: Rectal Cancer with Disseminated Carcinomatosis of the Bone Marrow

\section{References}

1 Singh G, Krause JR, Breitfeld V. Bone marrow examination: for metastatic tumor: aspirate and biopsy. Cancer. 1977;40(5):2317-21.

2 Rhee J, Han SW, Oh DY, Im A, Kim TY, Bang YJ. Clinicopathologic features and clinical outcomes of gastric cancer that initially presents with disseminated intravascular coagulation: a retrospective study. J Gastroenterol Hepatol. 2010;25(9):1537-42.

3 Ringenberg QS, Doll DC, Yarbro JW, Perry MC. Tumors of unknown origin in the bone marrow. Arch Intern Med. 1986;146(10):2027-8.

4 Sugarbaker EV, Thornthwaite J, Ketcham AS. Inhibitory effect of a primary tumor on metastasis. In: Delrio G, Brachet J, editors. Progress in cancer research and therapy. New York, NY: Raven Press; 1997. pp. 227-40.

5 Jarcho S. Diffusely infiltrative carcinoma. A hitherto undescribed correlation of several varieties of tumor metastasis. Arch Pathol. 1936;22:674-96.

6 Brain MC, Azzopardi G, Baker LR, Pineo GF, Roberts PD, Dacie JV. Microangiopathic haemolytic anaemia and mucin-forming adenocarcinoma. Br J Haematol. 1970;18(2):183-93.

7 Hayashi H, Haruyama H, Emura Y, Kaizuka I, Ozeki T. Disseminated carcinomatosis of the bone marrow - Study of a type of metastatic cancer and relationship of microangiopathic hemolytic anemia or disseminated intravascular coagulation. Jpn J Cancer Clin. 1979;25:329 (in Japanese).

8 Yoshioka K, Shimizu H, Yokoo S, Andachi H. Disseminated carcinomatosis of bone marrow from submucosal carcinoma in adenoma of the rectum. Intern Med. 1992;31(8):1056-9.

9 Huang WT, Chang KC, Shan YS, Tsao CJ, Lee JC. Successful initial treatment with weekly 24-hour infusion of 5 -fluorouracil and leucovorin in a rectal cancer patient with acute disseminated intravascular coagulation. Hepatogastroenterology. 2005;52(65):1436-9.

10 Misawa R, Kobayashi M, Ito M, Kato M, Uchikawa Y, Takagi S. Primary colonic signet ring cell carcinoma presenting carcinocythemia:an autopsy case. Case Rep Gastroenterol. 2008;2:301-7.

11 Naito M, Yoshida Y, Aisu N, Tanimura S, Hoshino S, Tanaka T, et al. A report of disseminated carcinomatosis of the bone marrow originating from transverse colon cancer successfully treated with chemotherapy using XELOX plus bevacizumab. Case Rep Oncol. 2014;7(2):426-34.

12 Nakashima Y, Takeishi K, Guntani A, Tsujita E, Yoshinaga K, Matsuyama A, et al. Rectal cancer with disseminated carcinomatosis of the bone marrow: report of a case. Int Surg. 2014;99(5):518-22.

13 van Bunderen CC, de Weger VA, Griffioen-Keijzer A. Disseminated intravascular coagulation as clinical manifestation of colorectal cancer: a case report and review of the literature. Neth J Med. 2014;72(4):186-9.

14 Lim DH, Lee SI, Park KW. Bone marrow metastasis of colon cancer as the first site of recurrence:a case report. Oncol Lett. 2014;8(6):2672-4.

15 Hanamura F, Shibata Y, Shirakawa T, Kuwayama M, Oda H, Ariyama H, et al. Favorable control of advanced colon adenocarcinoma with severe bone marrow metastasis:a case report. Mol Clin Oncol. 2016;5(5):579-82.

16 Takeyama H, Sakiyama T, Wakasa T, Kitani K, Inoue K, Kato H, et al. Disseminated carcinomatosis of the bone marrow with disseminated intravascular coagulation as the first symptom of recurrent rectal cancer successfully treated with chemotherapy: a case report and review of the literature. Oncol Lett. 2017;13(6):4290-4.

17 Nakamura S, Fukui T, Suzuki S, Takeda H, Watanabe K, Yoshioka T. Long-term survival after a favorable response to anti-EGFR antibody plus chemotherapy to treat bone marrow metastasis:a case report of KRASwildtype rectal cancer. Onco Targets Ther. 2017;10:1143-7.

18 Suenaga M, Mizunuma N, Matsusaka S, Shinozaki E, Ozaka M, Ogura M, et al. Phase II study of reintroduction of oxaliplatin for advanced colorectal cancer in patients previously treated with oxaliplatin and irinotecan: RE-OPEN study. Drug Des Devel Ther. 2015;9:3099-108.

19 Santini D, Vincenzi B, Addeo R, Garufi C, Masi G, Scartozzi M, et al. Cetuximab rechallenge in metastatic colorectal cancer patients: how to come away from acquired resistance? Ann Oncol. 2012;23(9):2313-8.

20 Liu X, George GC, Tsimberidou AM, Naing A, Wheler JJ, Kopetz S, et al. Retreatment with anti-EGFR based therapies in metastatic colorectal cancer: impact of intervening time interval and prior anti-EGFR response. BMC Cancer. 2015;15:713.

21 Shitara K, Yamanaka T, Denda T, Tsuji Y, Shinozaki K, Komatsu Y, et al. REVERCE: a randomized phase II study of regorafenib followed by cetuximab versus the reverse sequence for previously treated metastatic colorectal cancer patients. Ann Oncol. 2019;30:259-65.

22 Napolitano S, Martini G, Rinaldi B, Martinelli E, Donniacuo M, Berrino L, et al. Primary and acquired resistance of colorectal cancer to anti-EGFR monoclonal antibody can be overcome by combined treatment of regorafenib with cetuximab. Clin Cancer Res. 2015;21:2975-83. 